\title{
Performance Evaluation of Antlion Optimizer Based Regulator in Automatic Generation Control of Interconnected Power System
}

\author{
Esha Gupta and Akash Saxena \\ Department of Electrical Engineering, Swami Keshvanand Institute of Technology, Management and Gramothan, \\ Office No. AC-201, Ramnagaria, Jagatpura, Jaipur, Rajasthan 302017, India
}

Correspondence should be addressed to Esha Gupta; esha.gupta@outlook.com

Received 26 November 2015; Accepted 4 May 2016

Academic Editor: Chung-Liang Chang

Copyright (C) 2016 E. Gupta and A. Saxena. This is an open access article distributed under the Creative Commons Attribution License, which permits unrestricted use, distribution, and reproduction in any medium, provided the original work is properly cited.

This paper presents an application of the recently introduced Antlion Optimizer (ALO) to find the parameters of primary governor loop of thermal generators for successful Automatic Generation Control (AGC) of two-area interconnected power system. Two standard objective functions, Integral Square Error (ISE) and Integral Time Absolute Error (ITAE), have been employed to carry out this parameter estimation process. The problem is transformed in optimization problem to obtain integral gains, speed regulation, and frequency sensitivity coefficient for both areas. The comparison of the regulator performance obtained from ALO is carried out with Genetic Algorithm (GA), Particle Swarm Optimization (PSO), and Gravitational Search Algorithm (GSA) based regulators. Different types of perturbations and load changes are incorporated to establish the efficacy of the obtained design. It is observed that ALO outperforms all three optimization methods for this real problem. The optimization performance of ALO is compared with other algorithms on the basis of standard deviations in the values of parameters and objective functions.

\section{Introduction}

With the increase in the interconnection of the utilities, complexity in power system operation and control has emerged as a challenging problem in front of design engineers. Variation of the system parameters (voltage and frequency) from their nominal values can present a potential threat to the system stability. To control these deviations, intelligent design is required at generation, transmission, and distribution end. Hence, Automatic Generation Control (AGC) of interconnected power network is a major thrust area of research. To keep frequency and tie-line power exchanges in a nominal range AGC of the interconnected power system is required $[1,2]$. The prime objective of the successful power system operation is to transmit, distribute, and utilize the electrical power within the nominal range of frequency and terminal voltage. Normally, the interconnection of different power plants (nuclear, thermal, and hydro) introduces different complexities in the operation of power system. Hydro power plants are less operative in developing countries like India, as the availability of the water for irrigation purpose is a critical issue. Moreover, the constraints related to regulations hinder the participation of hydro power plants in the AGC. High efficiency of nuclear units prevents the participation of these units in AGC. The role of AGC is prominent in thermal power plants. Control of generator consists in functioning of two major loops: Automatic Voltage Regulator (AVR) loop to maintain the nominal voltage and Load Frequency Control (LFC) loop to maintain the system frequency.

In 1970, the concept of modern optimal control was introduced by Elgerd [1]. In the modern optimal control, the determination of the parameters of primary governor loop is performed to enhance the system's damping performance. In recent years, this field has emerged as a potential area of research. Approaches employed for AGC can be subdivided into two categories. First, the application of supervised learning models and expert systems was employed to obtain an intelligent control of interconnected power system. Second, the applications of metaheuristic techniques to obtain the controller settings were explored. A rich literature survey on 
AGC is provided in [3]. A philosophy of AGC is explained in $[4,5]$. Some of the approaches for effective AGC were based on Pole Placement Technique [6], Coefficient Diagram Method (CDM) [7, 8], Neural Networks (NN) [9-11], Fuzzy Logic (FL) [12-15], and Super Magnetic Energy Storage (SMES) Devices [16]. Calculation of CDM coefficients for large interconnected units involves heavy computations. These approaches require large data sets and observations for training and rule formation. Moreover, fuzzy approaches are based on approximations. AGC of a power system is a responsible operation; minute changes in the controller settings can put a question mark on the reliable operation of the power system. Hence, Neural Network and Fuzzy Logic based approaches are not suitable in modern power system's context. As "nature is the best teacher," by mimicking the biological behavior of plants, insects, and species, some beautiful analogies were developed and simulated by researchers in the form of mathematical paradigms. Some of these approaches include Gravitational Search Algorithm (GSA) [17] based on Newton's law of attraction, Particle Swarm Optimization (PSO) [18] based on the behavior of flock and swarm fishes, Genetic Algorithm (GA) [19] based on Darwinian's survival of the fittest and natural evolution, Bacterial Foraging (BF) [20] based on foraging behavior of bacteria, Differential Evolution (DE) [21], Artificial Bee Colony (ABC) [22] based on the behavior of bees, Firefly Algorithm (FA) [23], and Cuckoo Search (CS) [24]. Some hybrid approaches are also reported in which the Fuzzy Logic is combined with the PI controllers. Majorly, the proportional and integral gains of a controller were considered as parameters of interest in the optimization process. However, in the literature, parameters of primary governor loop were also optimized in [20]. The effect of speed regulation on the performance of the regulator was also discussed in [25]. Recently, Teaching Learning Based Optimization (TLBO) is applied to find the scaling factors and integral gains for two thermal units' interconnected power systems in [26]. Recently, Grey Wolf Optimizer (GWO) is applied to find out the optimal settings of PID controller for three thermal units by Sharma and Saikia [27]. In the work, frequency droop was observed in the presence of solar power plants. Bat algorithm is applied to find the regulator settings of multiarea thermal power system in [28]. The author employed PD-PID cascade controllers to obtain the AGC. From the literature review, it is clear that application of metaheuristic algorithm in AGC regulator design is a potential area. The search of a proper set of parameters (integral and differential gains, primary loop parameters) by which Area Control Error (ACE) can be reduced to zero is a major objective to solve AGC problem. In the literature, two objective functions (design criteria) were employed to carry out the estimation process. To find the minima of these functions by considering the gains and other parameters as variables is the essence of AGC problem. Evolutionary algorithms search for global optima of the function by the combined actions of agents and deciding operator in a predefined search space. Hence, the quality of exploration and exploitation is a major deciding factor in the performance of the algorithm. Another noteworthy feature of these algorithms is randomness; although randomness gives different results in each run, they can yet be able to avoid the local minima trap.

Recently, Mirjalili proposed an Antlion Optimizer (ALO) algorithm on the behavior inspired from antlions [29]. ALO has been successfully applied over 19 benchmark functions along with four classical engineering problems. This algorithm is based on the foraging behavior of antlions. Moreover, salient features of algorithms are the effective exploration of the search space by random walk and random selection of agents. Similarly, exploitation of the search space is assured by adaptive boundaries of traps. Since it is a population based algorithm, the avoidance of local optima is indispensable. Fewer parameters, gradient-free structure, and adaptive intensity with iterations are some salient features of the algorithm. In view of the above literature survey, salient features and computational efficacy of the ALO motivated us to employ ALO in AGC regulator design for the very first time. The following are the objectives of this research work:

(1) To solve the optimization process by ISE and ITAE objective functions to find out the parameters of primary governor loop, that is, speed regulation constant $(R)$, frequency bias $(D)$, and integral gains $\left(K_{I}\right)$.

(2) To test the efficacy of the objective functions with the help of damping performance obtained by ALO regulators.

(3) To test the robustness and efficacy of the proposed design with other recently employed regulators and test the design for various types of perturbations and topological changes.

This paper is organized as follows. The details of system modeling are presented in brief in Section 2. In Section 3, details of ALO along with the functioning of operators are explained in a lucid manner. Section 4 discusses the simulation results and analysis. Section 5 provides the comparative analysis of the optimization process for all the algorithms. And finally, in Section 6, conclusions and future scope of the work are pointed out.

\section{System Modeling}

2.1. AGC Model. The two-area nonreheat thermal interconnected power system is shown in Figure 1. The main components of the power system include speed governor, turbine, rotating mass, and load. The inputs of the power system are controller output $u$, load disturbance $\Delta P_{L}$, and tie-line power $\Delta P_{\text {tie }}$ and the outputs are frequency deviations $\Delta f$ and Area Control Error (ACE). The ACE signal controls the steady state errors of frequency deviation and tie-power deviation. Mathematically, ACE can be defined as

$$
\mathrm{ACE}=B \Delta f+\Delta P_{\text {tie }},
$$

where $B$ indicates the frequency bias parameter.

The operating behavior of the power system is dynamic so it must be assumed that the parameters of the system are linear. For mathematical modeling, the transfer function is used. 


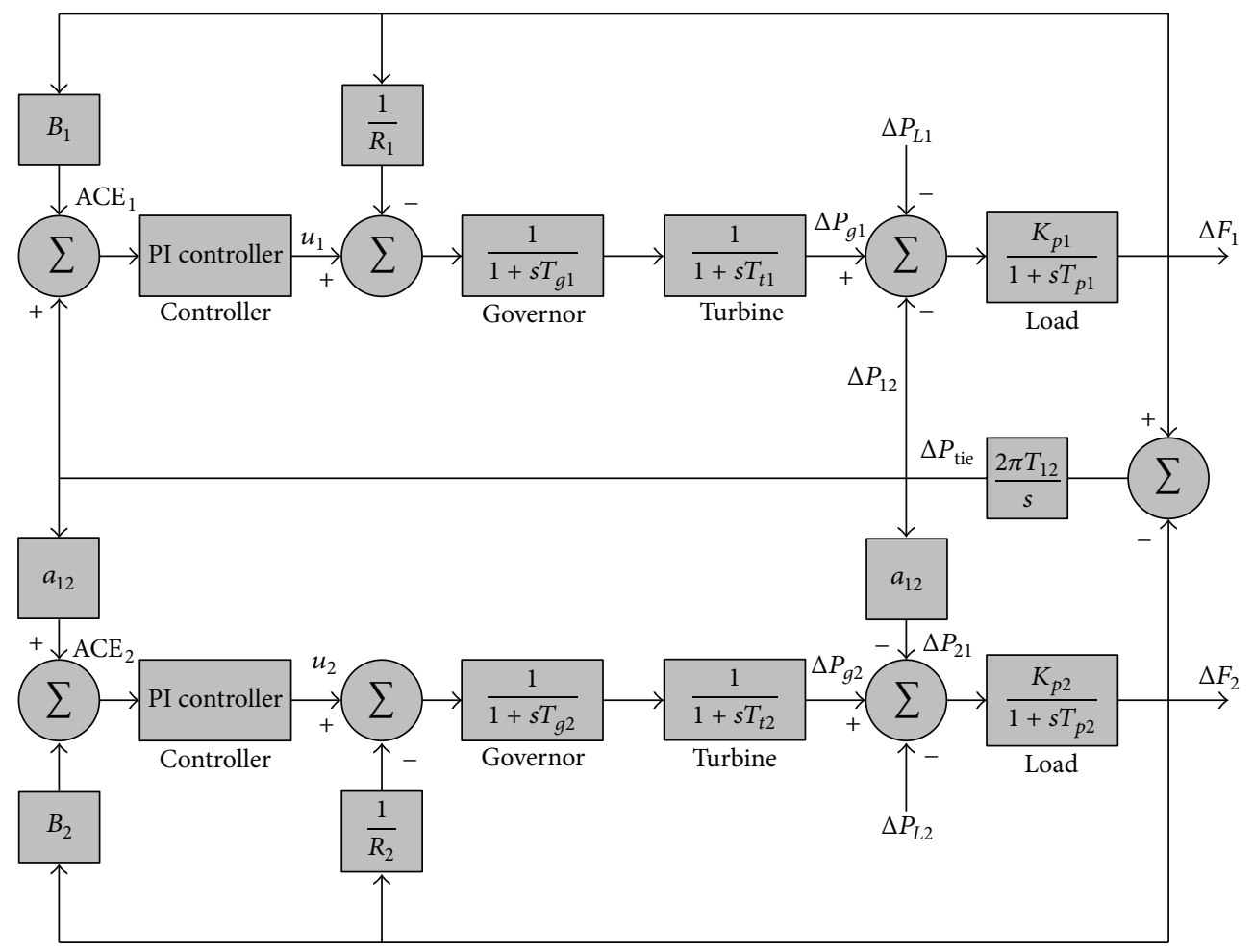

FIGURE 1: Transfer function model of two-area nonreheat thermal interconnected system.

The transfer function of a governor is represented by [1]

$$
G_{g}(s)=\frac{1}{1+s T_{g}} .
$$

The turbine is represented by the transfer function as [1]

$$
G_{t}(s)=\frac{1}{1+s T_{t}} .
$$

The transfer function of rotating mass and load [1] is as follows:

$$
G_{L}(s)=\frac{K_{p}}{1+s T_{p}},
$$

where $T_{p}=2 H / f D$ and $K_{p}=1 / D$.

$\Delta P_{G}$ and $\Delta P_{L}$ are the two inputs of rotating mass and load and $\Delta f(s)$ is the output and is represented by [1]

$$
\Delta f(s)=G_{L}(s)\left[\Delta P_{G}(s)-\Delta P_{L}(s)\right] .
$$

2.2. The System Investigated. The system was investigated on two equal thermal areas connected by a weak tie line having the same generation capacity of $1000 \mathrm{MVA}$. The parameters of the system are taken from [4]. A sudden step perturbation of 0.1875 p.u. occurs in area 1 and another one of 0.1275 p.u. occurs in area 2. The transfer function model of the twoarea thermal system is shown in Figure 1. The system is implemented using MATLAB 2013 and run on a Pentium IV CPU, 2.69 GHz, and 1.84 GB RAM computer [30].
2.3. The Proposed Approach. The controller used in AGC system is PI controller as it determines the difference between set point and reference point and removes the steady state error. For the design of PI controller, the parameters proportional gain $\left(K_{P}\right)$ and integral gain $\left(K_{I}\right)$ are essential. However, in this work, for the ease and simplicity of the optimization process, we consider proportional gain 1. Area Control Errors are the input of the controllers for area 1 and area 2 and are defined as

$$
\begin{aligned}
& \mathrm{ACE}_{1}=B_{1} \Delta f_{1}+\Delta P_{\text {tie }} \\
& \mathrm{ACE}_{2}=B_{2} \Delta f_{2}+\Delta P_{\text {tie }}
\end{aligned}
$$

where $B_{1}=1 / R_{1}+D_{1}$ and $B_{2}=1 / R_{2}+D_{2}$.

The outputs of the controllers are $u_{1}$ and $u_{2}$ and are obtained as follows:

$$
\begin{aligned}
& u_{1}=K_{P 1} \mathrm{ACE}_{1}+K_{I 1} \int \mathrm{ACE}_{1}, \\
& u_{2}=K_{P 2} \mathrm{ACE}_{2}+K_{I 2} \int \mathrm{ACE}_{2} .
\end{aligned}
$$

In this paper, the estimation of integral gains and parameters of primary governor loop is based on two objective functions (ITAE and ISE) which are given in (8). These objective 
functions aim to reduce the steady state error to zero and maximize the damping ratio of the system. Hence,

$$
\begin{aligned}
& J_{1}=\operatorname{ITAE}=\int_{0}^{T}\left(\left|\Delta f_{1}\right|+\left|\Delta f_{2}\right|+\left|\Delta P_{\text {tie }}\right|\right) \cdot t d t, \\
& J_{2}=\operatorname{ISE}=\int_{0}^{T}\left(\left|\Delta f_{1}\right|^{2}+\left|\Delta f_{2}\right|^{2}+\left|\Delta P_{\text {tie }}\right|^{2}\right) d t .
\end{aligned}
$$

The problematic constraints are the parameters of AGC regulator which contains integral gains, speed regulations, and the frequency sensitivity coefficients as they are bounded with the limits. These parameters are system specific. Hence, the design problem can be formulated as follows:

$$
\begin{aligned}
\text { Minimize } & J \\
\text { Subjected to } & K_{I_{\min }} \leq K_{I} \leq K_{I_{\max }} \\
& R_{\min } \leq R \leq R_{\max } \\
& D_{\min } \leq D \leq D_{\max } .
\end{aligned}
$$

$J$ is the objective function $\left(J_{1}\right.$ and $\left.J_{2}\right)$.

\section{Antlion Optimizer}

A novel algorithm inspired by nature named Antlion Optimizer (ALO) is presented in this section. This technique was proposed by Mirjalili [29] in 2015. In ALO, the hunting mechanism of antlions is mimicked. Antlions belong to Myrmeleontidae family of class net winged insects. ALO employs five main steps of hunting, that is, random walk of ants, building trap, entrapment of ants in trap, catching prey, and rebuilding traps. The ALO algorithm is a gradientfree algorithm which also provides greater exploration and exploitation of search space. Exploration is guaranteed by the random selection of antlions and random walks of ants around them whereas exploitation is guaranteed by adaptive shrinking boundaries of antlion's trap. With the help of roulette wheel and random walks, ALO has high probability to resolve local optima stagnation. The life cycle of antlions consists of two main phases: larvae and adults. Total natural lifespan can take up to 3 years which mostly occurs in larvae and only 3-5 weeks in adulthood. Antlions undergo metamorphosis in a cocoon to become adult. They mostly hunt in larvae and the adulthood period is for reproduction. An antlion larva digs a cone shaped pit in sand by moving along a circular path and throwing out sand with its massive jaw. After digging the trap, the larvae hide underneath the bottom of the cone and wait for the insect (preferably ant) to be trapped in the pit. The edge of the cone is sharp enough for insects to fall to the bottom of the trap easily. Figure 2 illustrates the hunting behavior in which antlions wait for the ants to be trapped in the cone shaped pit.

Once the antlion realizes that the prey is in the trap, it tries to catch it. Another interesting behavior in the lifestyle of ant behavior is the relevancy of size of the trap, level of hunger, and shape of the moon. Antlions dig out larger traps as they become more hungry and when the moon is full. And in this way they improve their chance of survival.
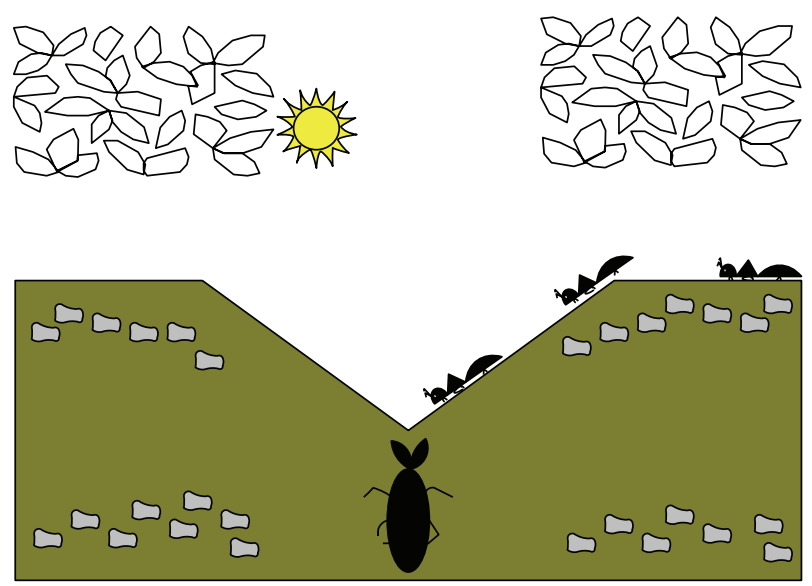

FIGURE 2: The hunting behavior of antlion.

\subsection{Mathematical Modeling of the ALO Algorithm}

(a) Random Walks of Ants. Random walks of ants are given in

$$
\begin{aligned}
& X(t)=\left[0, \operatorname{cumsum}\left(2 r\left(t_{1}\right)-1\right),\right. \\
& \left.\quad \operatorname{cumsum}\left(2 r\left(t_{2}\right)-1\right), \ldots, \operatorname{cumsum}\left(2 r\left(t_{n}\right)-1\right)\right],
\end{aligned}
$$

where $n$ is the maximum number of iterations, cumsum calculates the cumulative sum, and $t$ is the step of random walk. Hence,

$$
r(t)= \begin{cases}1 & \text { if rand }>0.5 \\ 0 & \text { if } \text { rand }<0.5 .\end{cases}
$$

Here, $r(t)$ is a stochastic function and rand is a random number generated with uniform distribution in the interval of $[0,1]$.

The positions of ants are saved and utilized during optimization in the matrix:

$$
M_{\text {Ant }}=\left[\begin{array}{ccccc}
A_{1,1} & A_{1,2} & \cdots & \cdots & A_{1, d} \\
A_{2,1} & A_{2,2} & \cdots & \cdots & A_{2, d} \\
\vdots & \vdots & \vdots & \vdots & \vdots \\
\vdots & \vdots & \vdots & \vdots & \vdots \\
A_{n, 1} & A_{n, 2} & \cdots & \cdots & A_{n, d}
\end{array}\right]
$$

where $M_{\text {Ant }}$ is the matrix for saving the position of each ant, $A_{i, j}$ shows the value of the $j$ th variable of $i$ th ant, $n$ is the number of ants, and $d$ is the number of variables.

At each step of optimization, ants update their position according to random walk. Equation (10) cannot be directly used for updating position of ants. The random walks are normalized using the following equation (min-max normalization):

$$
X_{i}^{t}=\frac{\left(X_{i}^{t}-a_{i}\right) \times\left(d_{i}-c_{i}^{t}\right)}{\left(d_{i}^{t}-a_{i}\right)}+c_{i}
$$


TABLE 1: Optimized parameters of AGC regulator.

\begin{tabular}{lcccccccc}
\hline \multirow{2}{*}{ Parameters } & \multicolumn{2}{c}{ ALO } & \multicolumn{2}{c}{ GSA [17] } & \multicolumn{2}{c}{ PSO [18] } & $J_{1}$ & $J_{1}$ \\
& $J_{1}$ & $J_{2}$ & $J_{1}$ & $J_{2}$ & $J_{2}$ & 0.3031 & 0.6525 \\
$K_{I 1}$ & 0.3260 & 0.4002 & 0.3817 & 0.4171 & 0.3131 & 0.4498 & 0.31 \\
$K_{I 2}$ & 0.2135 & 0.2010 & 0.2153 & 0.2028 & 0.1091 & 0.2158 & 0.3063 & 0.7960 \\
$R_{1}$ & 0.0491 & 0.0404 & 0.0401 & 0.0435 & 0.0581 & 0.0201 & 0.0794 & 0.0503 \\
$R_{2}$ & 0.0699 & 0.0509 & 0.0657 & 0.0635 & 0.0531 & 0.03 & 0.0737 & 0.0609 \\
$D_{1}$ & 0.4457 & 0.4884 & 0.5889 & 0.4778 & 0.4756 & 0.5910 & 0.7591 & 0.7216 \\
$D_{2}$ & 0.8770 & 0.8975 & 0.8946 & 0.8744 & 0.6097 & 0.8226 & 0.8950 & 0.8984 \\
\hline
\end{tabular}

where $a_{i}$ is the minimum of random walk of $i$ th variable, $d_{i}$ is the maximum of random walk of $i$ th variable, $c_{i}^{t}$ is the minimum of $i$ th variable at $t$ th iteration, and $d_{i}^{t}$ is the maximum of $i$ th variable at $t$ th iteration.

(b) Trapping in Antlion's Pit. Random walks of ants are affected by antlions' trap. Mathematical modeling of trapping in antlion's pit is proposed by the following equations:

$$
\begin{aligned}
& c_{i}^{t}=\text { Antlion }_{j}^{t}+c^{t}, \\
& d_{i}^{t}=\text { Antlion }_{j}^{t}+d^{t},
\end{aligned}
$$

where $c^{t}$ represents the minimum of all variables at $t$ th iteration, $d^{t}$ indicates the vector including the maximum of all variables at $t$ th iteration, $c_{i}^{t}$ is the minimum of all variables for $i$ th ant, $d_{i}^{t}$ is the maximum of all variables for $i$ th ant, and Antlion $_{j}^{t}$ shows the position of the selected $j$ th antlion at $t$ th iteration.

(c) Building Trap. For building trap, a roulette wheel is employed to model the hunting capability of antlions. The ALO algorithm is required to utilize a roulette wheel operator for selecting antlions based on their fitness during optimization. This mechanism provides high chances to the fitter antlions for catching ants.

(d) Sliding Ants towards Antlion. Antlions are able to build traps which are proportional to their fitness and ants are required to move randomly. Once the antlion realizes that an ant is in the trap, it shoots sand out the centre of the pit. The ants which are trying to escape slide down the trap. The radius of the ant's random walks hypersphere is decreased adaptively in the mathematical modeling. The following equations are proposed for this:

$$
\begin{aligned}
& c^{t}=\frac{c^{t}}{I}, \\
& d^{t}=\frac{d^{t}}{I},
\end{aligned}
$$

where $I$ is a ratio, $c^{t}$ is the minimum of all variables at $t$ th iteration, and $d^{t}$ indicates the vector including the maximum of all variables at $t$ th iteration.

(e) Catching Prey and Rebuilding the Pit. This is the final stage of hunt. At this stage, an ant reaches the bottom of the pit and is caught in the antlion's jaw. After this stage, the antlion pulls the ant inside the sand and consumes its body. Catching the prey occurs when the ant goes inside the sand and becomes fitter than its corresponding antlion. According to the position of the latest hunted ant, the antlions update their position to enhance the chances of catching new prey. Mathematically, the following equations can be proposed in this regard:

$$
\operatorname{Antlion}_{j}^{t}=\operatorname{Ant}_{i}^{t} \text { if } f\left(\operatorname{Ant}_{i}^{t}\right)>f\left(\text { Antlion }_{j}^{t}\right),
$$

where $t$ represents the current iteration, Antlion $_{j}^{t}$ is the position of the selected $j$ th antlion at $t$ th iteration, and $\mathrm{Ant}_{i}^{t}$ represents the position of $i$ th ant at $t$ th iteration.

(f) Elitism. For any evolutionary algorithm, elitism is an important feature that allows antlions to maintain the best solution obtained at any stage of optimization process. In this algorithm, the best obtained antlion during the entire iteration is saved and is considered as an elite. Since the fittest antlion is elite, it affects the movement of all the ants during iteration. Hence, it is assumed that every ant walks randomly around a selected antlion by roulette wheel and the elite simultaneously as follows:

$$
\mathrm{Ant}_{i}^{t}=\frac{R_{A}^{t}+R_{E}^{t}}{2},
$$

where $R_{A}^{t}$ is the random walk around the antlion selected by the roulette wheel at $t$ th iteration, $R_{E}^{t}$ is the random walk, and $\mathrm{Ant}_{i}^{t}$ represents the position of $i$ th ant at $t$ th iteration.

The following section presents analysis of simulation results.

\section{Results and Analysis}

This section presents simulation results and analysis of AGC regulator performance on two-area thermal interconnected power system with different step perturbations and loading conditions. Different AGC regulator settings are obtained with the application of four algorithms (GA, PSO, GSA, and ALO) on two standard objective functions (ISE and ITAE). Table 1 shows the values of optimized parameters of regulator with the application of the abovementioned algorithms on two objective functions.

Table 2 shows the values of system's minimum damping ratio and eigenvalues after the application of these AGC 


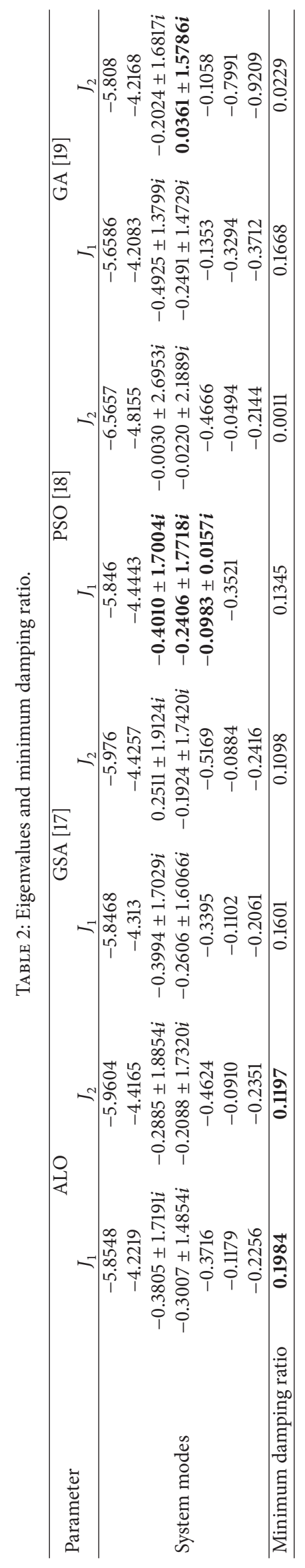


regulators. Eigenvalue analysis plays an important role in stability studies. Complex conjugate eigenvalues are also known as swing modes and these eigenvalues are responsible for oscillatory instability, when the real part of the eigenvalue is positive. From Table 2, it is observed that when optimization process is carried out with the application of GA on $J_{2}$ system mode contains a swing mode with positive eigenvalue (0.361). Real positive part of eigen is the indication of oscillations of growing amplitude. The minimum damping ratios obtained from the application of different regulators with $J_{1}$ and $J_{2}$ criteria are shown in Table 2. For PSO regulator, minimum damping ratios obtained from these criteria are $(0.1345,0.0011)$; similarly, the ratios for GA are $(0.1668,0.0229)$, for GSA are $(0.1601,0.1098)$, and for ALO are $(0.1984,0.1197)$. It can be said that a considerable amount of damping is enhanced in each case when the regulator parameters are obtained with criterion $J_{1}$. Overall damping of the system is the highest with ALO regulator $\left(J_{1}\right)(0.1984)$. Prima facie, it can be concluded that the regulator design obtained from criterion $J_{1}$ is more effective as the damping enhanced by this regulator is higher. In eigenvalue analysis, both real and imaginary parts have their interpretation and physical significance. The real part of the complex conjugate eigenvalue shows the damping behavior which represents the damp oscillations, which means the larger the magnitude, the higher the rate of decay. Imaginary components show the frequency of oscillations. It can be observed from Table 2 that high frequency oscillations are associated with setting $J_{2}$. Higher frequency oscillations are bad for equipment health and often cause the damage of physical structure of controllers. In this case for $J_{2}$, GA frequency of oscillations is $(1.57,1.68)$ for PSO, $(2.69,2.18)$ for GSA $(1.91,1.74)$, and $(1.88,1.73)$ for ALO. It is observed that although frequency of oscillations is in moderate range for GA regulator, the amplitude of the oscillation is growing with time as it has a positive real part of eigenvalue. However, the other regulators have high frequency of oscillations modes as compared with ALO. To show this analysis in a more prominent way, AGC regulators are designed with ALO algorithm and tested for different levels of perturbations. Figures 3(a) and 3(b) show the dynamic responses of frequency deviations in areas 1 and 2 when area 1 observes a step disturbance of 0.01 p.u. Figures $3(\mathrm{c})$ and $3(\mathrm{~d})$ show the frequency deviation curves of both areas with both regulator settings $J_{1}$ and $J_{2}$ when area 2 is perturbed with 0.02 p.u. Similarly, for both regulator settings, the dynamic responses obtained from both areas are self-explanatory. It is observed that $J_{1}$ setting is promising. The overshoot and settling time of the frequency deviation curves of both areas are less with $J_{1}$ regulator. It is also empirical to judge that the variations of tie-line power exchanges are nominal with both types of perturbation with $J_{1}$ regulator. Hence, it is concluded that $J_{1}$ optimization criterion is suitable for the designing of the AGC regulator.

To exhibit the comparative performance of the ALO regulator with other regulators, four different loading scenarios are simulated in this work. These loading conditions are summarized below.
Case 1. Load changes in area 1 by $10 \%$. The dynamic responses of $\Delta F_{1}, \Delta F_{2}$, and $\Delta P_{\text {tie }}$ are given in Figures 4-6 for all the algorithms.

Case 2. Load changes in area 2 by $20 \%$. Figures $7-9$ show the dynamic responses of the system.

Case 3. Load is increased in area 1 by $25 \%$. In Figures 10-12, the system dynamic responses are shown.

Case 4. Load is decreased in area 1 by $25 \%$ and the system dynamic responses are given in Figures 13-15.

Dynamic responses along with the system eigenvalues for these conditions are exhibited in Table 3 . It is observed that again with setting $J_{2}$ few eigenvalues possess positive real part when optimized with GA $(0.0370,0.0382$, and 0.0368$)$. The real part of swing mode varies from -0.2823 to -0.4567 for ALO regulator, from -0.2541 to -0.4632 for GSA regulator, from -0.0982 to -4.587 for PSO regulator, and from -0.2511 to -0.5411 for GA regulator with criterion $J_{1}$. It is of note here that the real part of the eigenvalue observes a large variation in case of GA under different loading conditions. This spread put a question mark on the performance of the regulator and robustness of the regulator also. Moderate spread has been observed with ALO regulator. For all cases, higher numeric values of real part of the eigenvalues suggest that the system is more stable. In Case 1, these values are $(-0.4278,-0.2823)$ for ALO, $(-0.4288,-0.2570)$ for GSA, $(-0.4277,-0.2395)$ for PSO, and $(-0.2588,-0.5271)$ for GA. It can be predicted that for Case 1 the robust setting is achieved by ALO. Similarly, in Case 4, the real parts of eigenvalues (swing modes) are $(-0.3276,-0.2879)$ for ALO and $(-0.3106,-0.2589)$ for GSA and an additional swing mode with PSO setting has been observed, $\left(J_{1}\right)(-0.3055,-0.2459,0.0983)$ and $(-0.440,0.2680)$ for GA. From this, it is also observed that a higher degree of robustness can be achieved by ALO regulator. To understand the dynamic response of the frequency deviation curves, a conventional index Figure of Demerit (FOD) is used in this paper. Figure of Demerit is the summation of the square of the overshoot and settling time of the deviation curves. It is observed that for almost all loading cases the values of settling time, overshoot, and FODs are low for ALO based regulators as compared with other regulator designs. It is observed from Figures 4-6 that ALO based controller exhibits better dynamic performance as compared with others. The percentage of overshoot and settling time is much less in these cases. The low oscillatory response exhibited by ALO is best suited for the equipment's health. FOD values are considered as a close replica of dynamic performance of controller. Higher values of FOD show poor dynamic performance and vice versa. It is also empirical to mention here that for frequency deviation in area 1 the settling time and FOD obtained from ALO are 3.8 and 14.44, respectively, whereas, from GSA, PSO, and GA, the settling time and FOD are 5.6, 5.0, and 4.9 and 31.36, 25, and 24.01, respectively. The frequency deviation in area 2 also shows that the values of settling time and FOD are less when ALO 


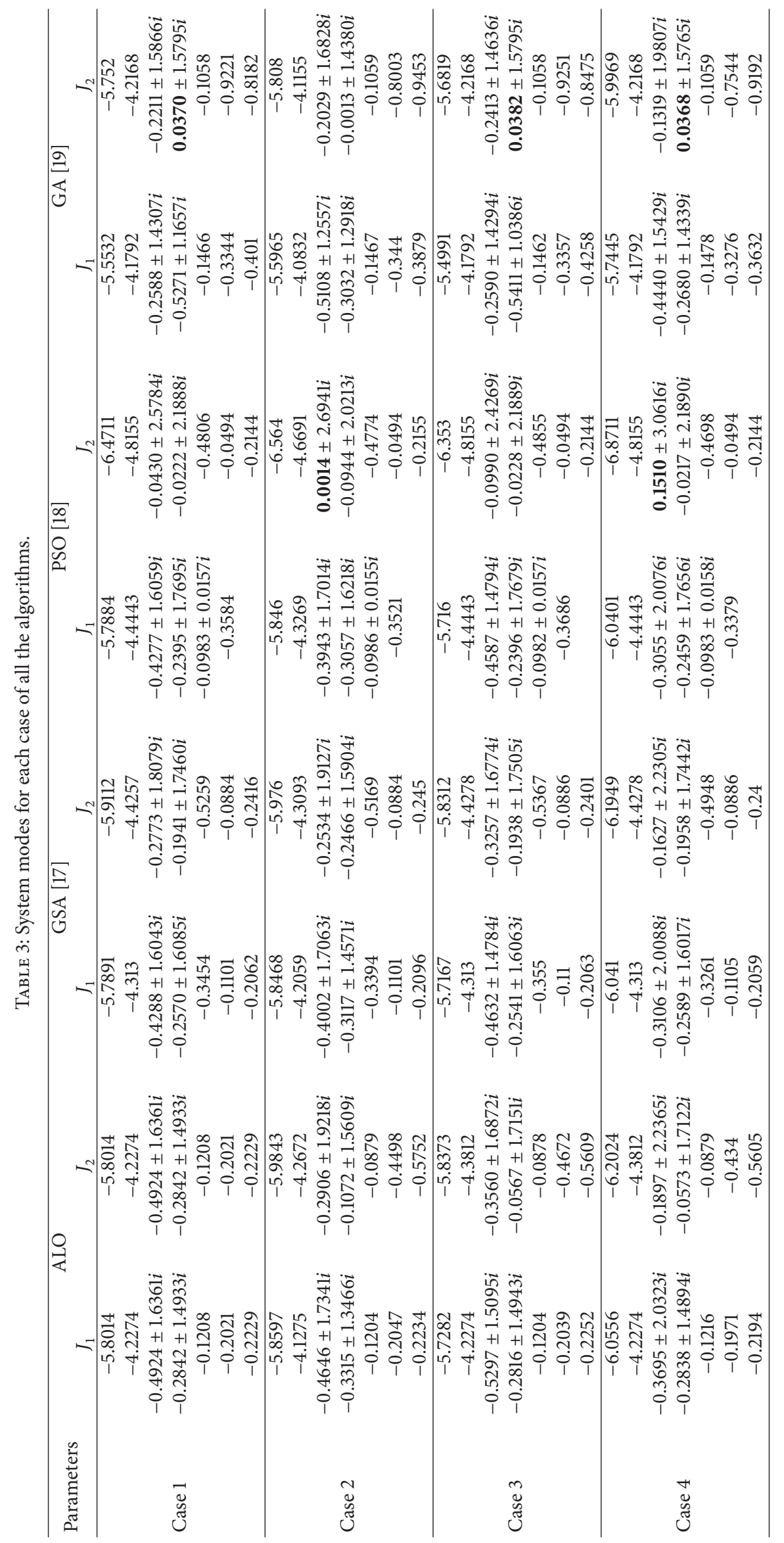




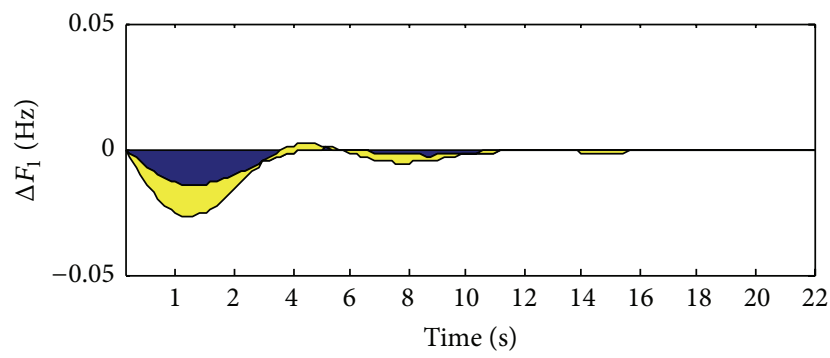

- $J_{1}$

$\square J_{2}$

(a)

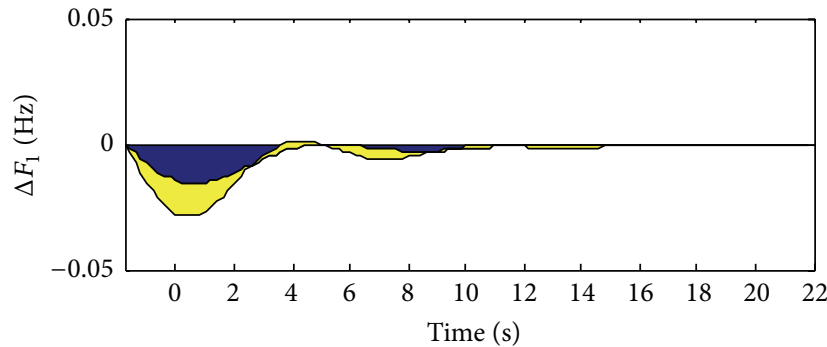

- $J_{1}$

$\square J_{2}$

(c)

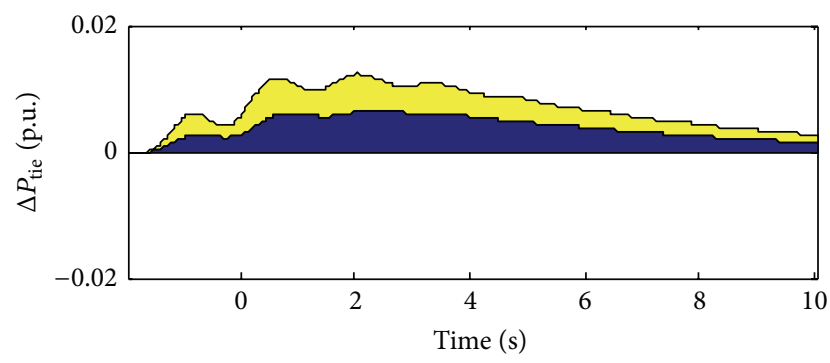

- $J_{1}$

$\square J_{2}$

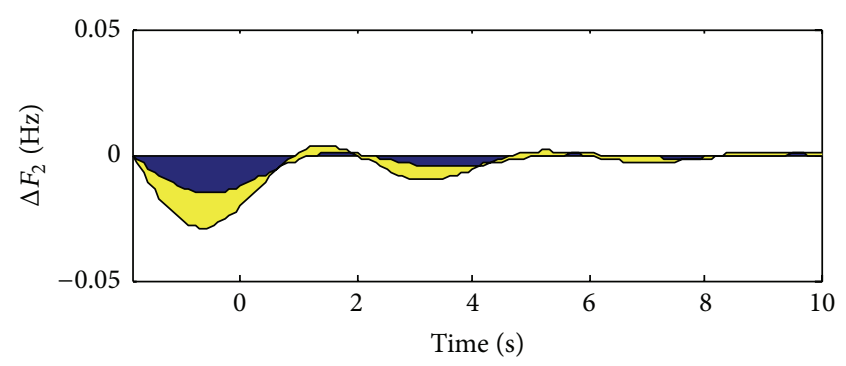

- $J_{1}$

$\square J_{2}$

(b)

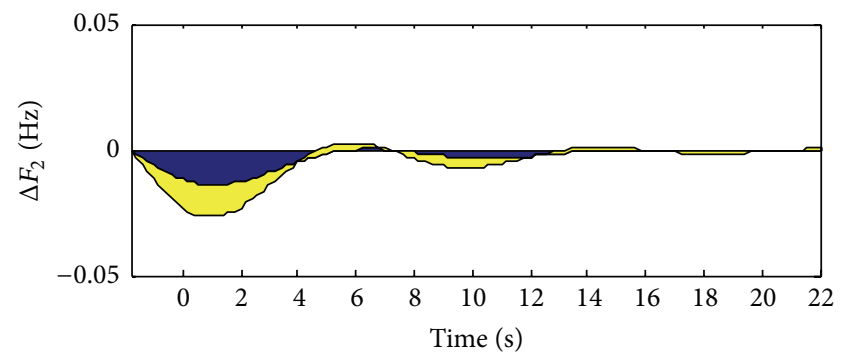

$\square J_{1}$

$\square J_{2}$

(d)

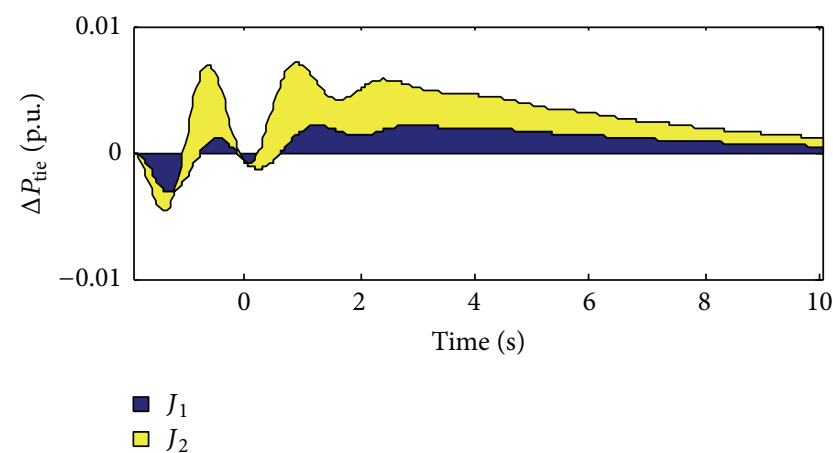

(f)

FIGURE 3: Dynamic responses obtained from ALO.

regulator is used. The value of settling time with frequency deviation in area 2 is observed as 6.0 for ALO, 7.2 for GSA, 7.6 for PSO, and 8.0 for GA. It is also interesting to observe that with the $10 \%$ increase in the load PSO gives erroneous results and the flow of tie-line power behaves in a different manner. Hence, critical analysis of dynamic responses clearly reveals that better dynamic performance is exhibited by ALO. By examining the responses in Figures 7-9, it is clearly seen that the settling time and peak overshoot are less when load changes in area 2 by $20 \%$. It can be observed from Figure 8 that when area 2 observes 20\% increase, GA based controller is not able to mitigate the frequency oscillations. This inculcates oscillatory instability in the system. However, ALO based controller shows a better dynamic response and yields satisfactory performance over a wide range of loading conditions. For Case 2, the settling time of ALO is 3.6 and is $5.3,4.2$, and 5.8 for GSA, PSO, and GA, respectively. Similarly, the FOD is also very low in case of ALO, that is, 12.96, whereas it is 17.64 and 33.64 in case of PSO and GA. Figures 10 and 11 show the frequency deviations of areas 1 and 2. From dynamic responses of overshoot, settling time, and FOD, it is clear that ALO provides competitive results. The dynamic responses for Case 4 are shown in Figures 13-15 and it has been observed that ALO tuned controller yields better dynamic performance. The minimum settling time and FOD obtained from ALO are 5.1 and 26.01 for frequency deviation in area 1 and 6.0 and 36.00 for frequency deviation in area 2. However, in case of GSA, the settling time is 7.9 and FOD is 19.8. An oscillatory response is obtained by the GA, GSA, and PSO tuned controllers. 


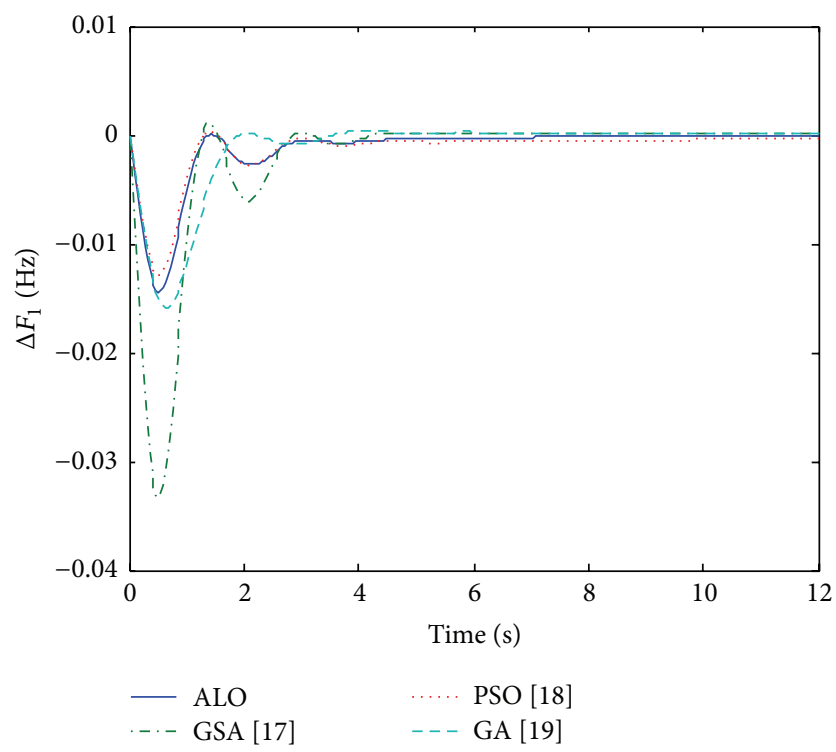

FIGURE 4: Change in frequency of area 1 by $10 \%$ load increase in area 1.

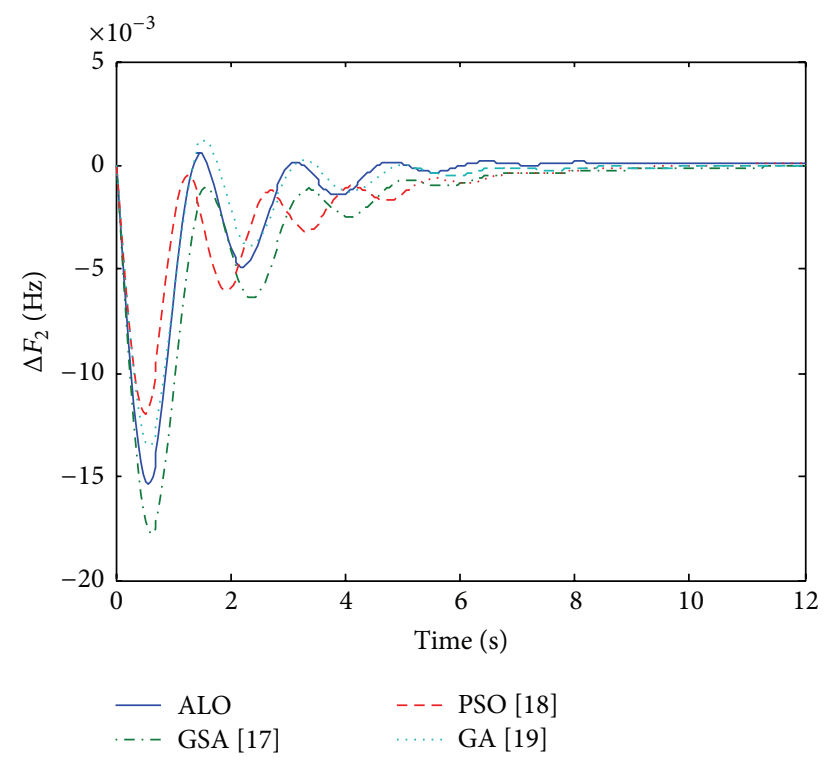

FIGURE 5: Change in frequency of area 2 by $10 \%$ load increase in area 1.

\section{Optimization Performance}

To judge the efficiency of the optimization process carried out by all algorithms, 100 trials of optimization are carried out. To provide a fair comparison, the population size (100) and the maximum number of iterations (1000) are kept the same. Stopping criterion for the optimization process is maximum run of the iteration. To observe the optimization process in a critical way, the standard deviations of optimized parameters of the regulator along with the values of objective functions are calculated and shown in Table 4 . It is observed that high values of standard deviations are obtained in regulator

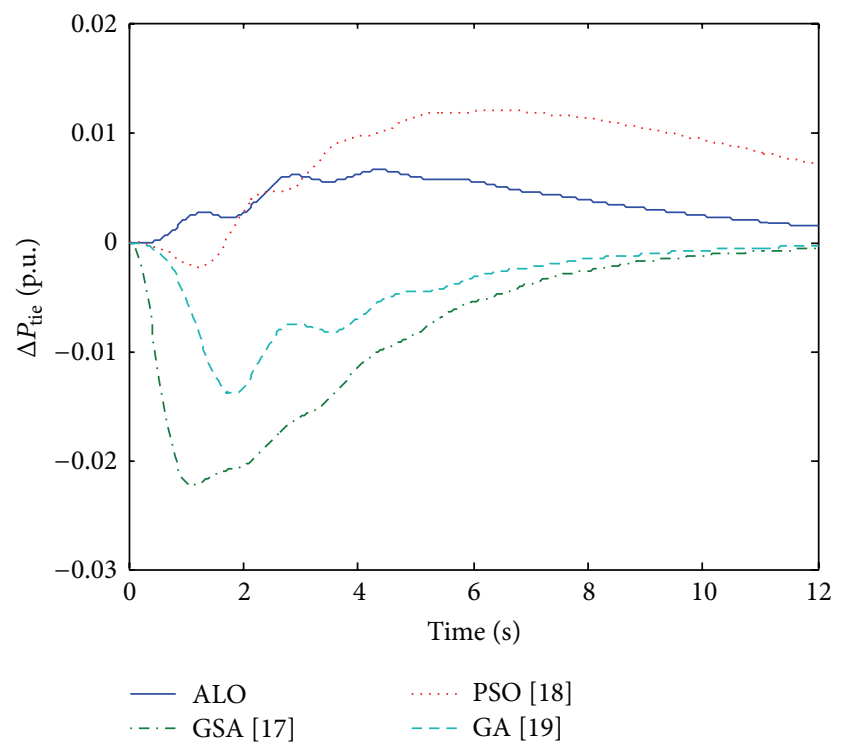

Figure 6: Change in tie-line power by $10 \%$ load increase in area 1.

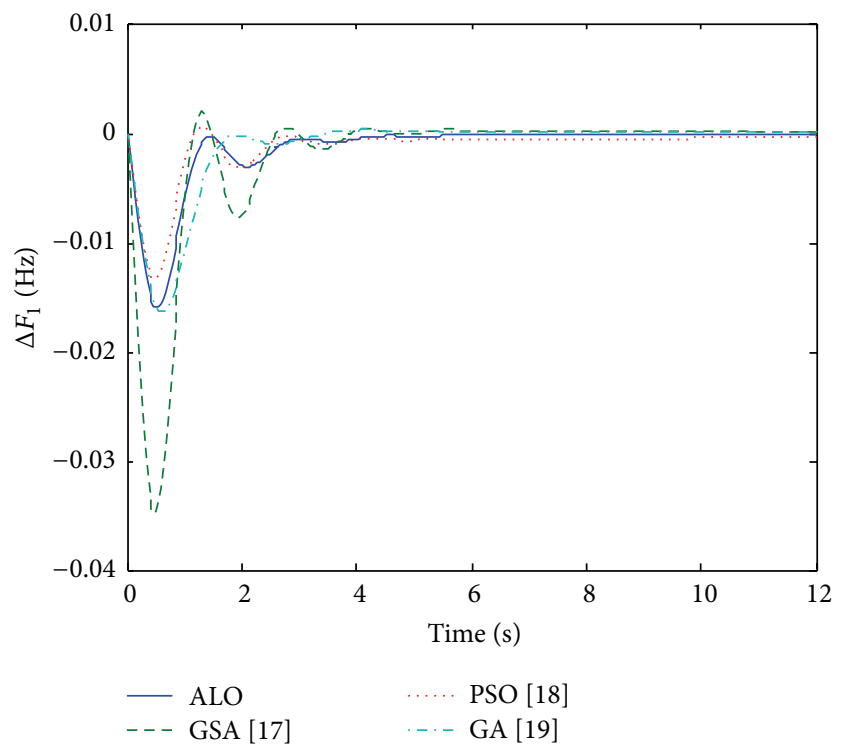

FIGURE 7: Change in frequency of area 1 by $20 \%$ load increase in area 2.

parameters and values of objective functions when optimization process is handled by GA (0.09729). Comparatively large values of standard deviations are found in GA and PSO when they are compared with ALO. We observed high values of standard deviation in speed regulation parameters after each run of optimization obtained with GA regulators $\left(J_{1}\right.$ and $J_{2}$ ). Speed regulation parameter is a vulnerable parameter in power system dynamics studies. It affects the system dynamics in a very prominent way. Large values of standard deviations in the calculation of such vulnerable parameters are not acceptable. The values for standard deviations are $0.10256\left(\mathrm{GSA}, J_{1}\right.$ ) and $0.1550\left(\mathrm{GA}, J_{2}\right)$ and, similarly, for $R_{2}$, they are 0.0081 (ALO, $\left.J_{1}\right), 0.02$ (GSA), 0.38 (PSO), and 
TABLE 4: Standard deviation of optimized parameters of the regulator.

\begin{tabular}{lcccccccc}
\hline \multirow{2}{*}{ Parameters } & \multicolumn{2}{c}{ ALO } & \multicolumn{2}{c}{ GSA [17] } & \multicolumn{2}{c}{ PSO [18] } & $J_{1}$ & $J_{1}$ \\
& $J_{1}$ & $J_{2}$ & $J_{1}$ & $J_{2}$ & $J_{1}$ & $J_{2}$ & 0.09729 \\
\hline$K_{I 1}$ & 0.02923 & 0.03773 & 0.02425 & 0.07720 & 0.02604 & 0.08461 & 0.20687 \\
$D_{1}$ & 0.04107 & 0.03315 & 0.04475 & 0.09833 & 0.08416 & 0.04759 & 0.18872 \\
$R_{1}$ & 0.00582 & 0.00102 & 0.00045 & 0.00168 & 0.00153 & 0.01403 & 0.00892 & 0.21313 \\
$K_{I 2}$ & 0.01763 & 0.04563 & 0.10256 & 0.00769 & 0.09639 & 0.08158 & 0.05800 & 0.15550 \\
$D_{2}$ & 0.08957 & 0.08807 & 0.04916 & 0.11771 & 0.16726 & 0.08363 & 0.17955 \\
$R_{2}$ & 0.00343 & 0.00174 & 0.00173 & 0.00385 & 0.00280 & 0.01080 & 0.00310 & 0.07945 \\
& 0.00184 & $7.16 E-06$ & 0.0212 & 0.000419 & 0.38 & 0.011 & 1.76 \\
\hline
\end{tabular}

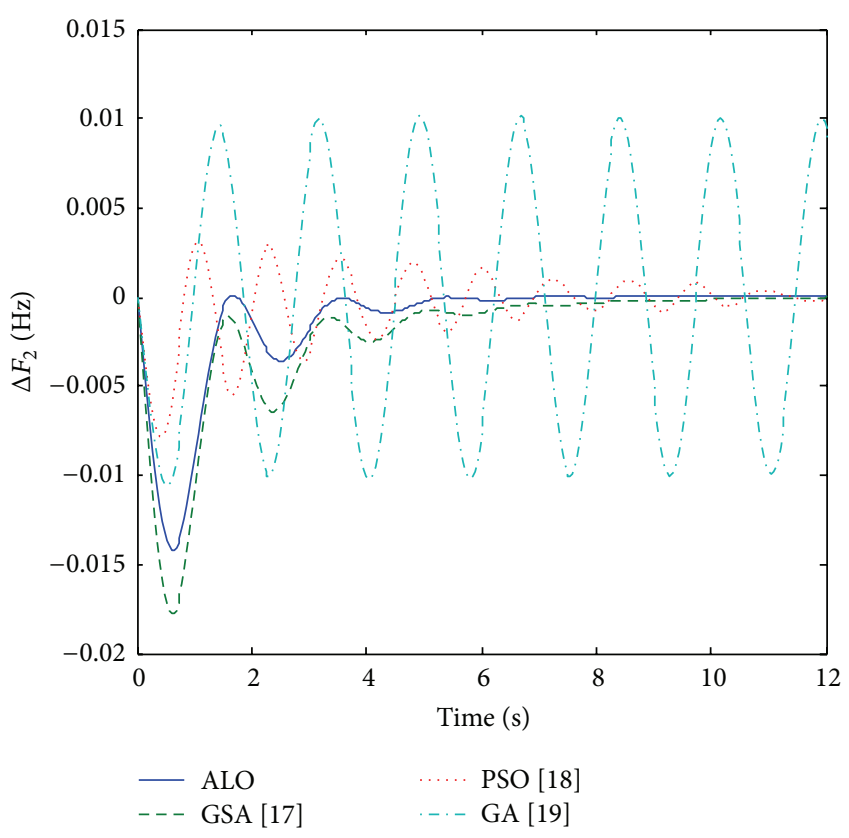

FIGURE 8: Change in frequency of area 2 by $20 \%$ load increase in area 2 .

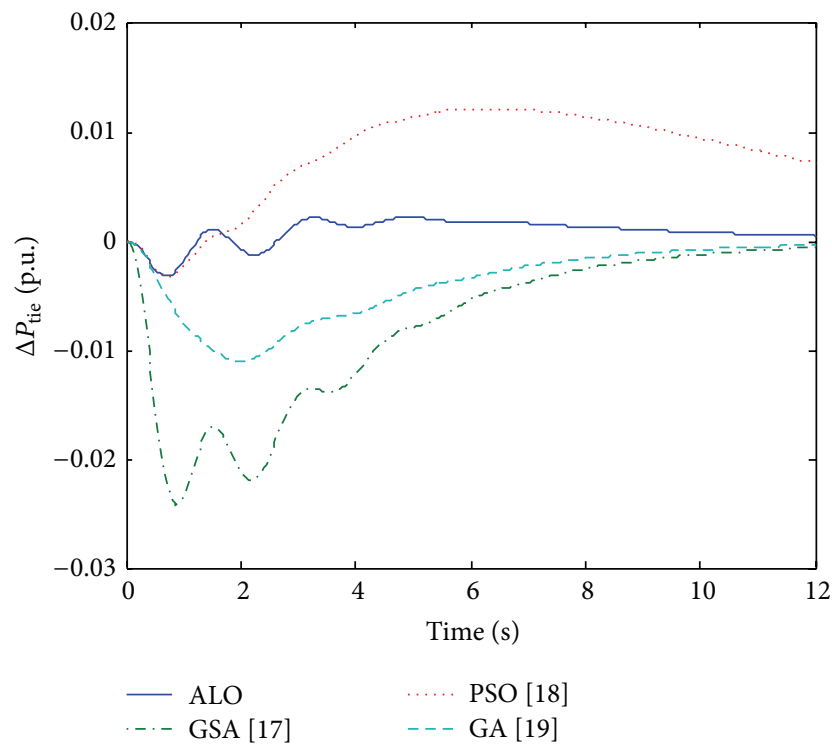

Figure 9: Change in tie-line power by $20 \%$ load increase in area 2.

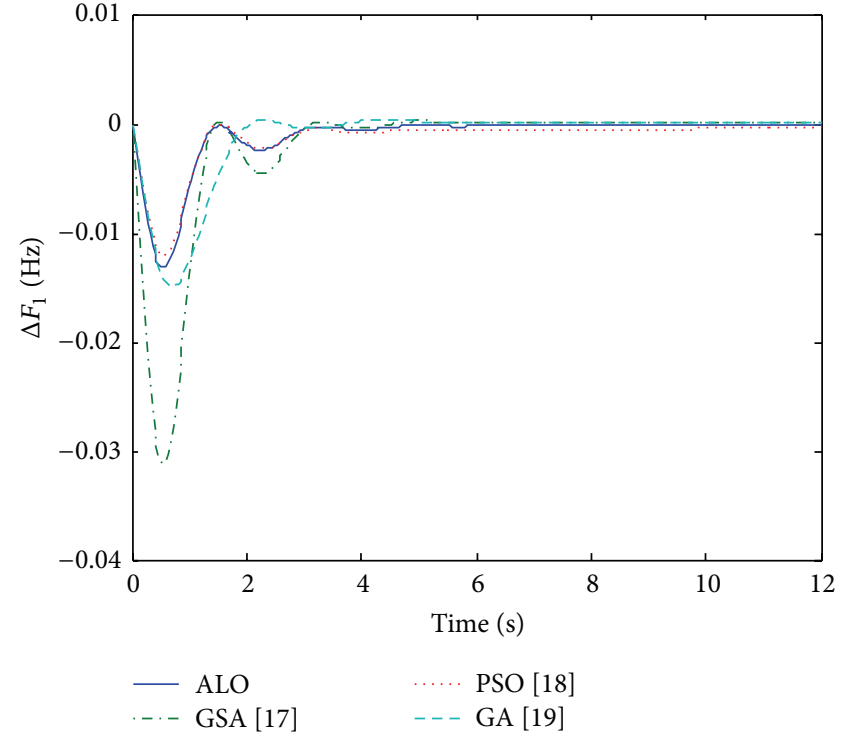

FIGURE 10: Change in frequency of area 1 by $25 \%$ load increase in area 1 .

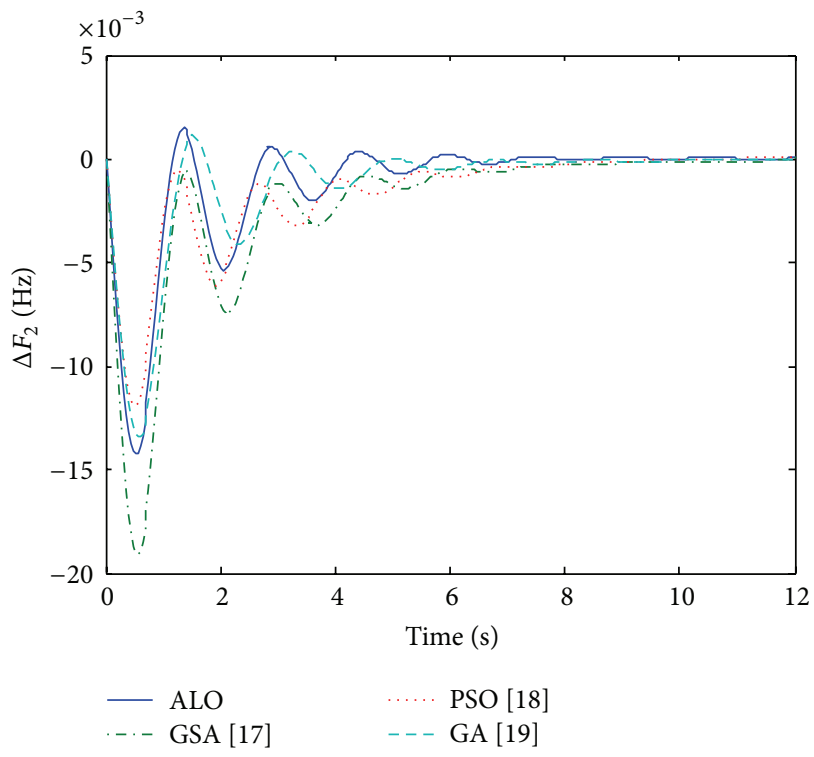

FIGURE 11: Change in frequency of area 2 by $25 \%$ load increase in area 1 . 


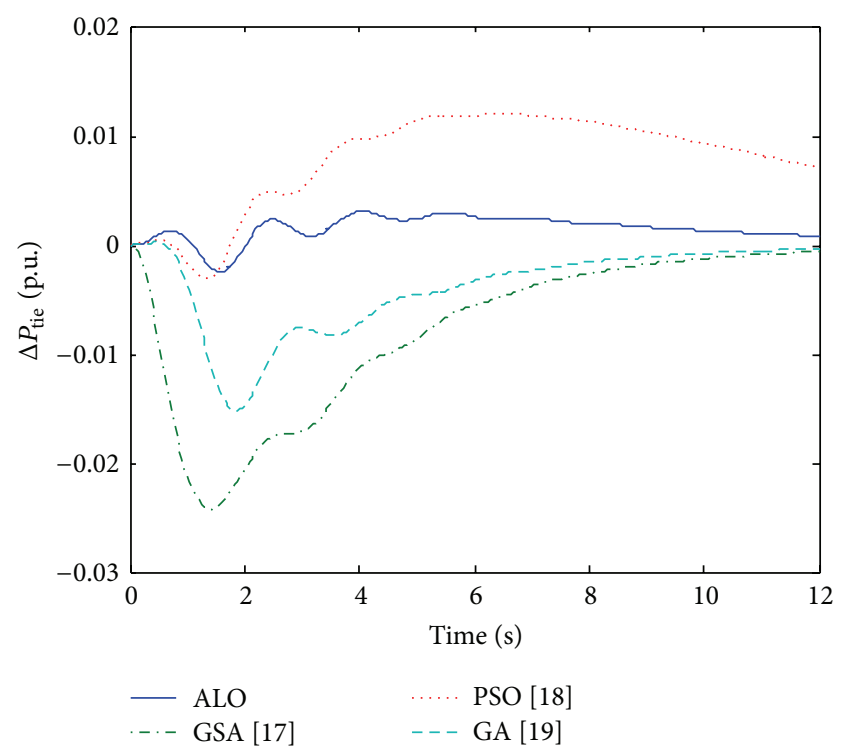

Figure 12: Change in tie-line power by $25 \%$ load increase in area 1.

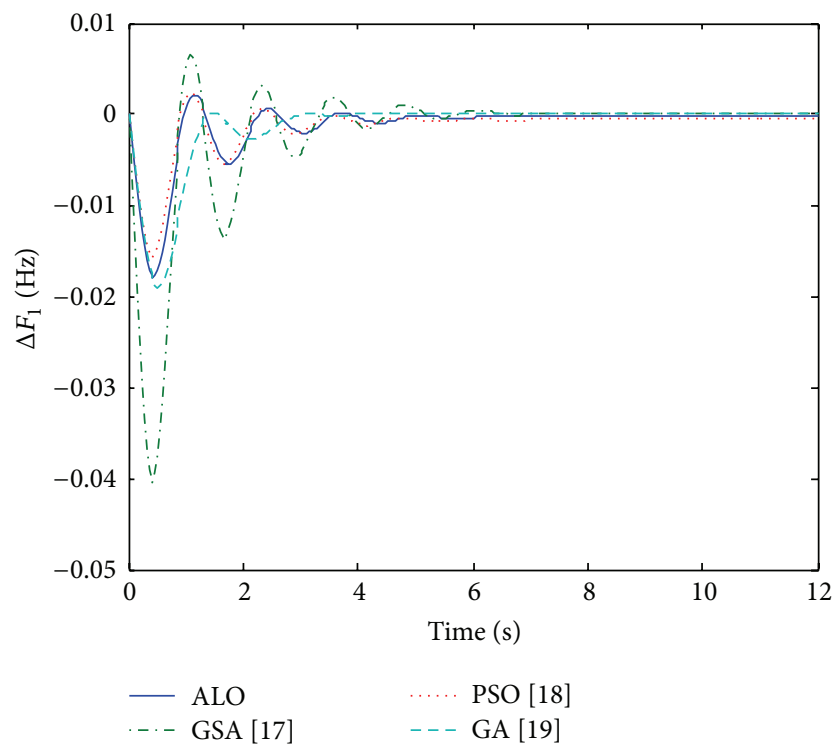

FIGURE 13: Change in frequency of area 1 by $25 \%$ load decreases in area 1.

1.76 for GA. The impact of speed regulation parameters on the dynamic response is shown in [25]. The lowest values of standard deviations are observed, when the parameters are optimized by ALO. This basically means that in each run of optimization ALO exhibits precision in computing the parameters. The standard deviations in the values of integral gains for area 1 by $J_{1}$ and $J_{2}$ are minimum for ALO (0.041, 0.033); for GA, these values are (0.18 and 0.213) and similarly $(0.044,0.09)$ for GSA and (0.08 and 0.047) for PSO. It can be concluded that regulator setting integral gain observes the least variation in numerical values when the parameter is optimized through $\operatorname{ALO}\left(J_{1}\right)$. The values of standard deviations in objective functions $J_{1}$ and $J_{2}$ are

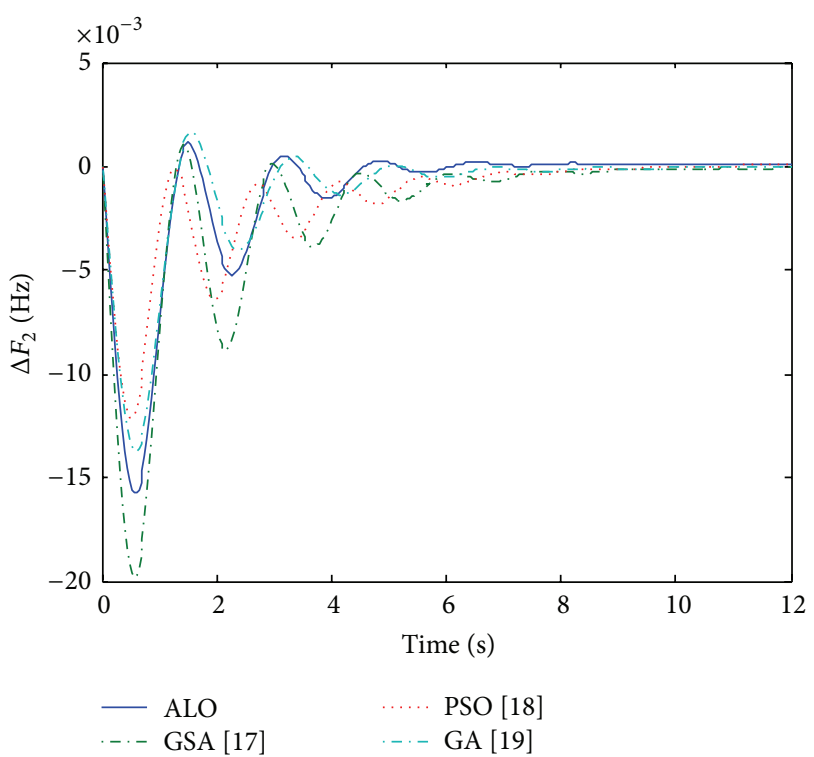

FIGURE 14: Change in frequency of area 1 by $25 \%$ load decreases in area 1 .

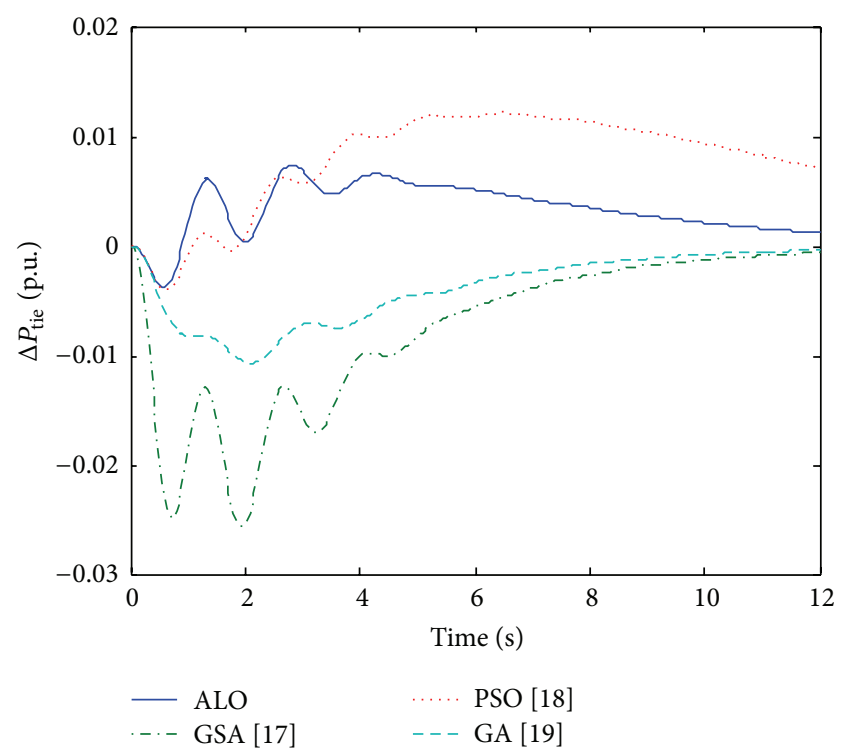

FIGURE 15: Change in tie-line power by $25 \%$ load decreases in area 1.

the lowest for ALO process and the highest for GA. The values of standard deviations in the values of $J_{1}$ for GA, PSO, GSA, and GWO are 0.0972, 0.0264, 0.0242, and 0.02 and for $J_{2}$ are $0.2068,0.0846,0.0772$, and 0.3773 . It has been observed that the values obtained by ALO are precise and the optimization processes are reliable enough for obtaining the regulator design. However, high values of standard deviations in parameters of regulator $\left(1.76\right.$ with $\left.R_{2}\right)$ and in the objective functions show that the optimization process loses its relevance when it is handled by GA. Hence, it is concluded from the eigenvalue analysis and dynamic response of the deviation curves that ALO shows a promising response to obtain regulator setting with optimization criterion $J_{1}$. The following 
section summarizes the contribution of this research work and proposes a solid milieu for future work.

\section{Conclusion}

This paper presents an application of the recently introduced algorithm ALO to find optimal parameters of the AGC regulator. The ALO regulator is employed on a test system of two thermal units connected with a weak tie line of limited capacity for AGC. The following are the major findings of the work:

(A) Comparison of the application of two objective functions, namely ISE and ITAE, in optimization process for finding the regulator parameters, under different contingencies, is investigated. Results reveal that ITAE is a better choice to optimize the regulator parameters.

(B) Eigenvalue analysis is performed to test the effectiveness of the proposed approach and to compare the results of the proposed approach with recently published approaches. It is observed that the damping obtained from ALO regulator is more positive as compared with the other algorithms.

(C) ALO shows promising results in terms of overshoot, settling time obtained from the frequency responses of both areas under different loading cases, standard deviations in regulator's parameter values, ISE and ITAE values, and optimization performance.

(D) Damping performance is evaluated with different contingencies, load changes, and step disturbances in both areas. PI controller setting obtained through ALO exhibits better dynamic performance and overall low settling time.

Application of other new metaheuristic algorithms in AGC regulator design on different models of the power system considering different renewable energy power sources lies in the future scope.

\section{Nomenclature}

$i$ : $\quad$ Subscript referring to area $i(1,2)$

$\Delta F_{i}: \quad$ Frequency deviation in area $i(\mathrm{~Hz})$

$\Delta P_{G i}$ : Incremental generation of area $i$ (p.u.)

$\Delta P_{L i}$ : Incremental load change in area $i$ (p.u.)

$\mathrm{ACE}_{i}$ : Area Control Error of area $i$

$B_{i}$ : $\quad$ Frequency bias parameter of area $i$

$R_{i}$ : $\quad$ Speed regulation of the governor of area $i$ (Hz/p.u. MW)

$T_{g i}: \quad$ Time constant of governor of area $i(\mathrm{~s})$

$T_{t i}: \quad$ Time constant of turbine of area $i(\mathrm{~s})$

$K_{p i}: \quad$ Gain of generator and load of area $i$

$T_{p i}:$ Time constant of generator and load of area $i(\mathrm{~s})$

$\Delta P_{\text {tie }}$ : Incremental change in tie line (p.u.)

$T_{12}$ : Synchronizing coefficient

T: $\quad$ Simulation time (s)

$t$ : Current iteration.

\section{Competing Interests}

The authors declare that they have no competing interests.

\section{References}

[1] O. I. Elgerd, Energy Systems Theory. An Introduction, McGrawHill, New Delhi, India, 1983.

[2] IEEE Standard Committee, "IEEE Standard definitions of terms for automatic generation control on electric power systems," IEEE Transactions on Power Apparatus and Systems, vol. 89, no. 6, pp. 1356-1364, 1970.

[3] A. Ibraheem, P. Kumar, and D. P. Kothari, "Recent philosophies of automatic generation control strategies in power systems," IEEE Transactions on Power Systems, vol. 20, no. 1, pp. 346-357, 2005.

[4] H. Sadat, Power System Analysis, McGraw-Hill, New York, NY, USA, 1999.

[5] J. Nanda and B. Kaul, "Automatic generation control of an interconnected power system," Proceedings of the Institution of Electrical Engineers, vol. 125, no. 5, pp. 385-390, 1978.

[6] A. Y. Sivaramakrishnan, M. V. Hariharan, and M. C. Srisailam, "Design of variable-structure load-frequency controller using pole assignment technique," International Journal of Control, vol. 40, no. 3, pp. 487-498, 1984.

[7] M. Z. Bernard, T. H. Mohamed, Y. S. Qudaih, and Y. Mitani, "Decentralized load frequency control in an interconnected power system using Coefficient Diagram Method," International Journal of Electrical Power and Energy Systems, vol. 63, pp. 165-172, 2014.

[8] R. Ali, T. H. Mohamed, Y. S. Qudaih, and Y. Mitani, "A new load frequency control approach in an isolated small power systems using coefficient diagram method," International Journal of Electrical Power and Energy Systems, vol. 56, pp. 110-116, 2014.

[9] I. Vajk, M. Vajta, L. Keviczky, R. Haber, J. Hetthéssy, and K. Kovács, "Adaptive load-frequency control of the hungarian power system," Automatica, vol. 21, no. 2, pp. 129-137, 1985.

[10] J. Kanniah, S. C. Tripathy, O. P. Malik, and G. S. Hope, "Microprocessor-based adaptive load-frequency control," IEE Proceedings C-Generation, Transmission and Distribution, vol. 131, no. 4, pp. 121-128, 1984.

[11] M. L. Kothari, P. S. Satsangi, and J. Nanda, "Sampled-data automatic generation control of interconnected reheat thermal systems considering generation rate constraints," IEEE Transactions on Power Apparatus and Systems, vol. 100, no. 5, pp. 23342342, 1981.

[12] S. Wu, M. J. Er, and Y. Gao, "A fast approach for automatic generation of fuzzy rules by generalized dynamic fuzzy neural networks," IEEE Transactions on Fuzzy Systems, vol. 9, no. 4, pp. 578-594, 2001.

[13] B. K. Sahu, S. Pati, P. K. Mohanty, and S. Panda, "Teachinglearning based optimization algorithm based fuzzy-PID controller for automatic generation control of multi-area power system," Applied Soft Computing Journal, vol. 27, pp. 240-249, 2015.

[14] K. R. Sudha and R. Vijaya Santhi, "Load frequency control of an interconnected reheat thermal system using type-2 fuzzy system including SMES units," International Journal of Electrical Power \& Energy Systems, vol. 43, no. 1, pp. 1383-1392, 2012.

[15] A. Banerjee, V. Mukherjee, and S. P. Ghoshal, "Intelligent controller for load-tracking performance of an autonomous 
power system," Ain Shams Engineering Journal, vol. 5, no. 4, pp. 1167-1176, 2014.

[16] S. Padhan, R. K. Sahu, and S. Panda, "Automatic generation control with thyristor controlled series compensator including superconducting magnetic energy storage units," Ain Shams Engineering Journal, vol. 5, no. 3, pp. 759-774, 2014.

[17] R. K. Sahu, S. Panda, and S. Padhan, "Optimal gravitational search algorithm for automatic generation control of interconnected power systems," Ain Shams Engineering Journal, vol. 5, no. 3, pp. 721-733, 2014.

[18] Y. L. Abdel-Magid and M. A. Abido, "AGC tuning of interconnected reheat thermal systems with particle swarm optimization," in Proceedings of the 10th IEEE International Conference on Electronics, Circuits and Systems (ICECS '03), pp. 376-379, December 2003.

[19] Y. L. Abdel-Magid and M. M. Dawoud, "Optimal AGC tuning with genetic algorithms," Electric Power Systems Research, vol. 38, no. 3, pp. 231-238, 1996.

[20] J. Nanda, S. Mishra, and L. C. Saikia, "Maiden application of bacterial foraging-based optimization technique in multiarea automatic generation control," IEEE Transactions on Power Systems, vol. 24, no. 2, pp. 602-609, 2009.

[21] U. K. Rout, R. K. Sahu, and S. Panda, "Design and analysis of differential evolution algorithm based automatic generation control for interconnected power system," Ain Shams Engineering Journal, vol. 4, no. 3, pp. 409-421, 2013.

[22] H. Gozde, M. C. Taplamacioglu, and I. Kocaarslan, "Comparative performance analysis of Artificial Bee Colony algorithm in automatic generation control for interconnected reheat thermal power system," International Journal of Electrical Power and Energy Systems, vol. 42, no. 1, pp. 167-178, 2012.

[23] S. Debbarma, L. Chandra Saikia, and N. Sinha, "Solution to automatic generation control problem using firefly algorithm optimized I $\lambda \mathrm{D} \mu$ controller," ISA Transactions, vol. 53, no. 2, pp. 358-366, 2014.

[24] P. Dash, L. C. Saikia, and N. Sinha, "Comparison of performances of several Cuckoo search algorithm based 2DOF controllers in AGC of multi-area thermal system," International Journal of Electrical Power and Energy Systems, vol. 55, pp. 429436, 2014.

[25] A. Saxena, M. Gupta, and V. Gupta, "Automatic generation control of two area interconnected power system using Genetic algorithm," in Proceedings of the 3rd IEEE International Conference on Computational Intelligence and Computing Research (ICCIC '12), Coimbatore, India, December 2012.

[26] B. K. Sahu, T. K. Pati, J. R. Nayak, S. Panda, and S. K. Kar, "A novel hybrid LUS-TLBO optimized fuzzy-PID controller for load frequency control of multi-source power system," International Journal of Electrical Power \& Energy Systems, vol. 74, pp. 58-69, 2016.

[27] Y. Sharma and L. C. Saikia, "Automatic generation control of a multi-area ST-thermal power system using Grey Wolf Optimizer algorithm based classical controllers," International Journal of Electrical Power and Energy Systems, vol. 73, pp. 853862, 2015.

[28] P. Dash, L. C. Saikia, and N. Sinha, "Automatic generation control of multi area thermal system using Bat algorithm optimized PD-PID cascade controller," International Journal of Electrical Power \& Energy Systems, vol. 68, pp. 364-372, 2015.

[29] S. Mirjalili, “The ant lion optimizer," Advances in Engineering Software, vol. 83, pp. 80-98, 2015.

[30] MATLAB, http://www.mathworks.com. 


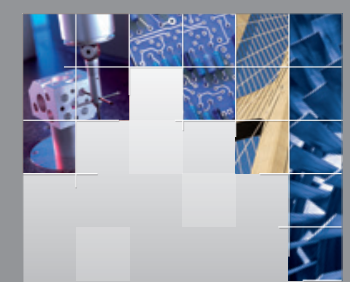

\section{Enfincering}
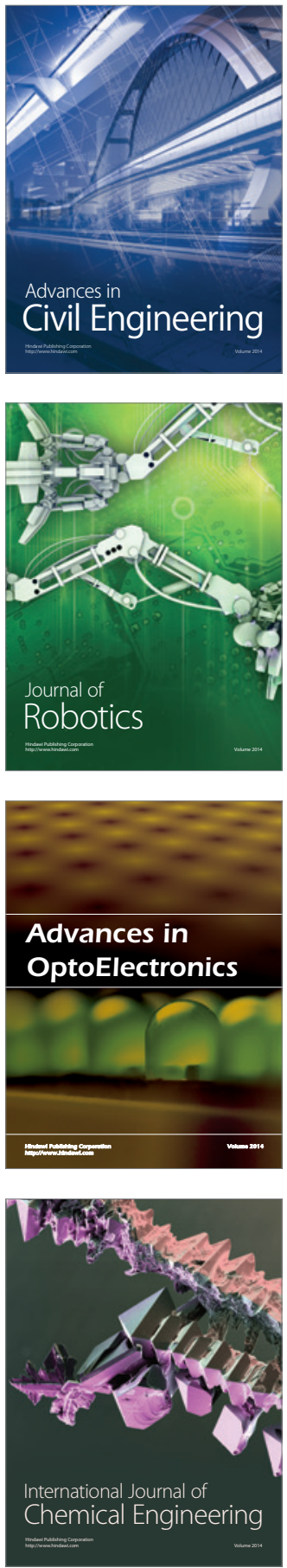

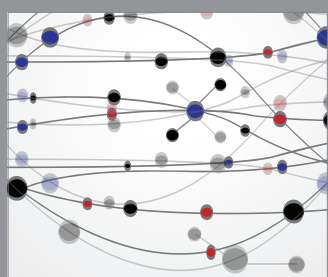

The Scientific World Journal

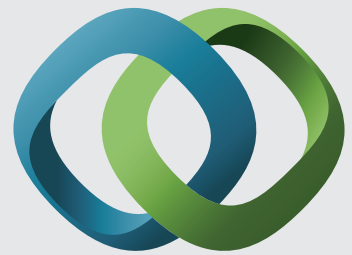

\section{Hindawi}

Submit your manuscripts at

http://www.hindawi.com
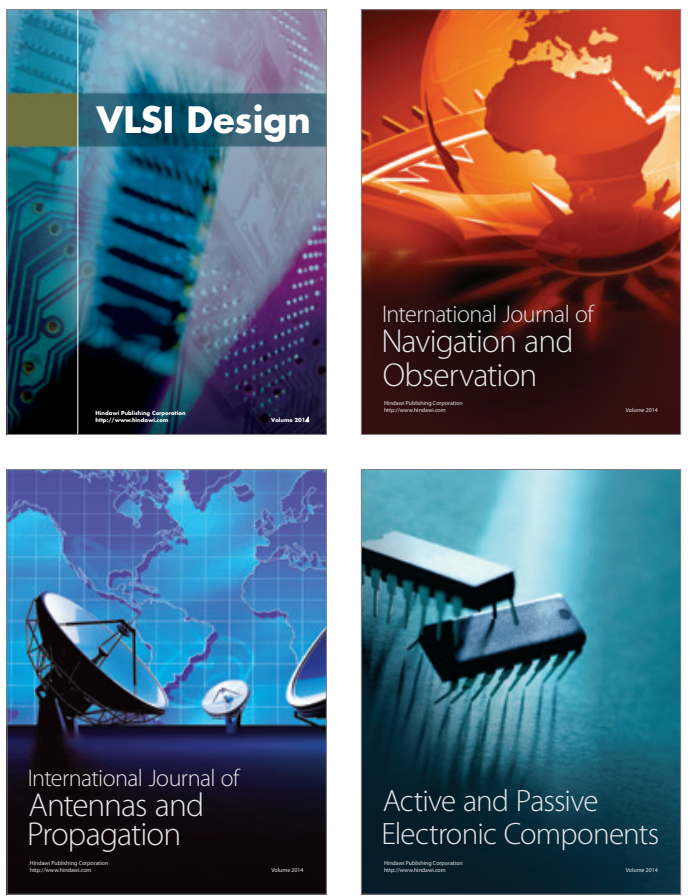
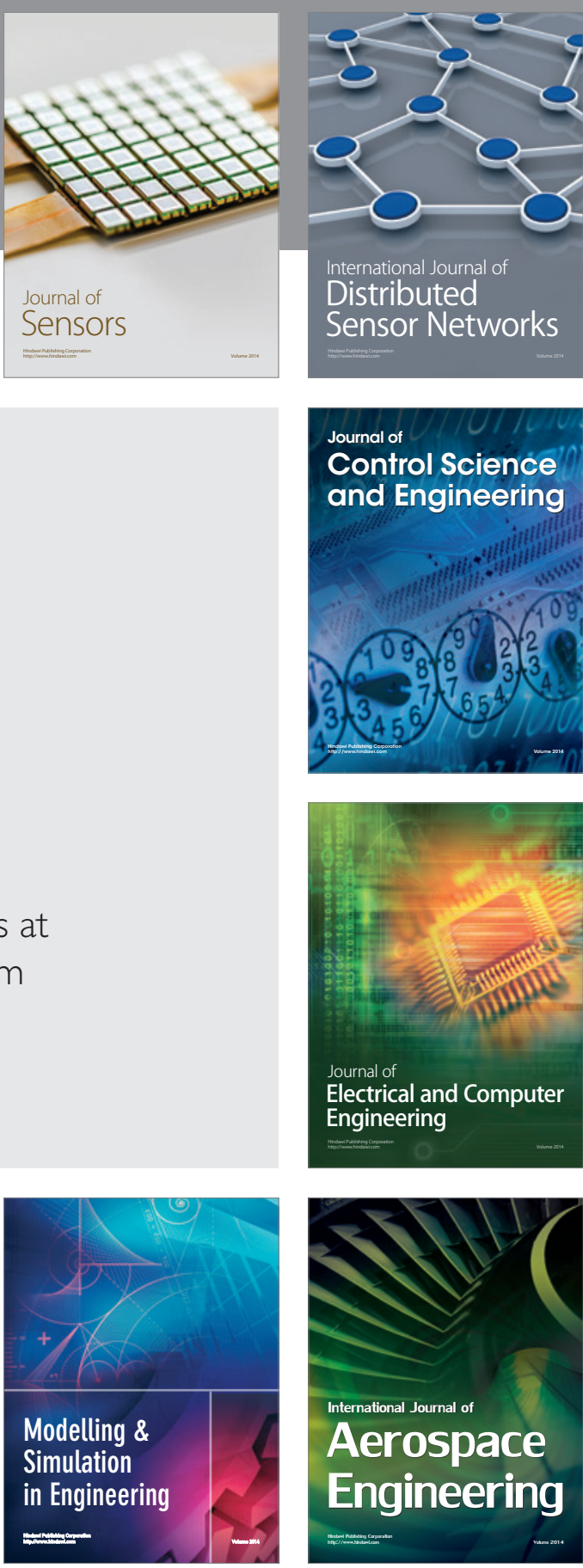

International Journal of

Distributed

Sensor Networks

Journal of

Control Science

and Engineering
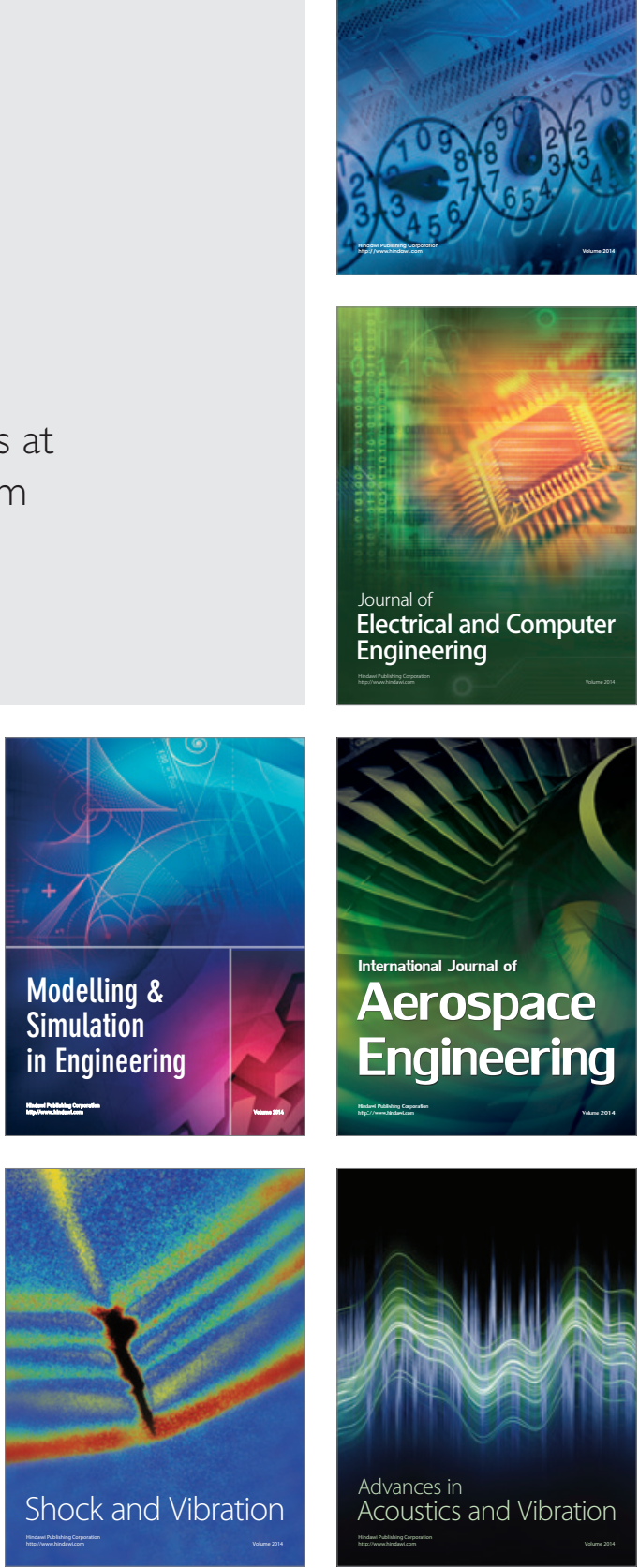